\title{
COMPUTER-ORIENTED REAL SPHERICAL HARMONICS FOR TEXTURE AND PROPERTIES ANALYSES
}

\author{
O. RAYMOND*, L. FUENTES** and J. I. GÓMEZ** \\ *Instituto Superior Pedagógico Enrique José Varona, Marianao, Habana, Cuba \\ **Instituto de Cibernética, Matemática y Física, Calle D \#353, \\ Habana 10400, Cuba
}

(Received 5 September 1995; in final form 5 March 1996)

An algorithm system to generate symmetric real functional bases for Quantitative Texture Analysis (QTA) is presented. A review of the analytical conditions to be satisfied by the considered functions is given. Suitable two- and three-dimensional bases are proposed. All crystal and sample point groups are analyzed. Computer implementation of the suggested algorithms is straightforward.

KEY WORDS: Spherical harmonics, real representation, crystal symmetry, sample symmetry.

\section{INTRODUCTION}

Spherical harmonics expansion of texture functions and polycrystal physical properties is a well-established technique. In this context Fourier functional bases provides a through system, although somewhat uncomfortable to use because of its complex character.

Another problem arises from the fact that, as a consequence of traditional interest in metal research (Bunge, 1982; Matthies, 1987), QTA algorithms for highly symmetrical crystal-sample combinations (such as centro symmetric cubic-orthorhombic) have already been studied in detail. However, for low-symmetry systems we have not found a systematic presentation of suitable functional bases.

Here we present a collection of explicit formulae and tables to generate two- and three-dimensional symmetric real functional bases for all crystal and sample point groups. Suggested functions follow all the mathematical and methodical precepts of Bunge's school. Computer implementation of the proposed algorithms is straightforward.

\section{THEORETICAL SUMMARY (for further details see Bunge, 1982)}

We briefly summarise some fundamental relations of QTA, as expressed with the aid of Bunge's spherical harmonics. Their fulfilment is to be considered as compulsory requirements for any alternative functional basis.

Orientation distribution function $f(\mathrm{~g})$ may be expanded under symmetry-adapted generalised spherical harmonics $\dot{\mathrm{T}}_{l}^{\mu \nu}(\mathrm{g})$ 


$$
f(\mathrm{~g})=\sum_{l=0}^{\infty} \sum_{\mu=1}^{M(l)} \sum_{v=1}^{N(l)} \mathrm{C}_{l}^{\mu \nu} \dot{\mathrm{T}}_{l}^{\mu \nu}(\mathrm{g})
$$

being

$$
\dot{\mathrm{T}}_{l}^{\mu \nu}(\mathrm{g})=\sum_{m=-1}^{l} \sum_{n=-l}^{l} \dot{\mathrm{A}}_{l}^{m \mu} \dot{\mathrm{A}}_{l}^{n v} \mathrm{~T}_{l}^{m n}(\mathrm{~g})
$$

The considered functional bases are linear combinations of the matrix elements of the irreducible representation of the three-dimensional rotation group $\mathrm{T}_{l}^{m n}(\mathrm{~g}$ ) (Gel'fand, Minlos and Shapiro, 1963). The functions $\dot{\mathrm{T}}_{l}^{\mu v}(\mathrm{~g})$ form an orthonormal basis fulfilling the condition

$$
\oint \ddot{\mathrm{T}}_{l}^{\mu v}(\mathrm{~g}) \ddot{\mathrm{T}}_{l^{*}}^{\mu^{\prime} v^{\prime}}(\mathrm{g}) \mathrm{dg}=\frac{1}{2 l+1} \delta_{l l^{\prime}} \delta_{\mu \mu^{\prime}} \delta_{v v^{\prime}}
$$

Likewise, Pole Figures and Inverse Pole Figures can be expanded as follows:

$$
\begin{aligned}
P_{\mathrm{h}_{i}}(\mathrm{y}) & =\sum_{l=0}^{\infty} \sum_{v=1}^{N(l)} F_{l}^{v}\left(\mathrm{~h}_{\mathrm{i}}\right) \dot{\mathrm{K}}_{l}^{v}(\mathrm{y}) \\
R_{\mathrm{y}_{i}}(\mathrm{~h}) & =\sum_{l=0}^{\infty} \sum_{\mu=1}^{M(l)} H_{l}^{\mu}\left(\mathrm{y}_{\mathrm{i}}\right) \dot{\mathrm{K}}_{l}^{* \mu}(\mathrm{h})
\end{aligned}
$$

The functions $\dot{\mathrm{K}}_{l}^{\mu}(\mathrm{h})\left[\dot{\mathrm{K}}_{l}^{v}(\mathrm{y})\right]$ are crystal [sample] symmetric spherical surface harmonics. They are represented by

$$
\begin{aligned}
\dot{\mathbf{K}}_{l}^{\mu}(\mathrm{h}) & =\sum_{m=-l}^{l} \dot{\dot{\mathrm{A}}}_{l}^{m \mu} \mathrm{K}_{l}^{m}(\mathbf{h}) \\
\dot{\mathrm{K}}_{l}^{v}(\mathrm{y}) & =\sum_{n=-l}^{l} \dot{\mathrm{A}}_{l}^{n v} \mathrm{~K}_{l}^{n}(\mathrm{y})
\end{aligned}
$$

where the initial spherical harmonics $\mathrm{K}_{l}^{m}(\mathrm{r})$ are explicitly defined by

$$
\mathrm{K}_{l}^{m}(\mathbf{r})=\mathrm{K}_{l}^{m}(\phi, \beta)=\frac{1}{\sqrt{2 \pi}} \overline{\mathrm{P}}_{l}^{m}(\phi) e^{i m \beta}
$$

with $\overline{\mathrm{P}}_{l}^{m}(\phi)$ being the normalised associated Legendre Polynomials.

The functions (6) and (7) satisfy the conditions:

$$
\oint \dot{\mathrm{K}}_{l}^{\mu}(\phi, \beta) \dot{\mathrm{K}}_{l^{\prime}}^{* \mu^{\prime}}(\phi, \beta) \sin \phi d \phi d \beta=\delta_{l l^{\prime}} \delta_{\mu \mu^{\prime}}
$$

Moreover, among (2), (6) and (7) the following relations hold:

$$
\dot{\mathrm{T}}_{l}^{\mu 0}(\mathrm{~g})=\dot{\mathrm{T}}_{l}^{\mu 0}\left(\phi, \varphi_{2}\right)=\sqrt{\frac{4 \pi}{2 l+1}} \dot{\mathrm{K}}_{l}^{\mu}\left(\phi, \varphi_{2}=\frac{\pi}{2}\right)=\sqrt{\frac{4 \pi}{2 l+1}} \dot{\mathrm{K}}_{l}^{* \mu}(\phi, \beta)
$$




$$
\dot{\mathrm{T}}_{l}^{0 v}(\mathrm{~g})=\dot{\mathrm{T}}_{l}^{0^{v v}}\left(\varphi_{1}, \phi\right)=\sqrt{\frac{4 \pi}{2 l+1}} \dot{\mathrm{K}}_{l}^{v}\left(\phi, \varphi_{1}=\frac{\pi}{2}\right)=\sqrt{\frac{4 \pi}{2 l+1}} \dot{\mathbf{K}}_{l}^{v}(\phi, \gamma)
$$

\section{REAL TWO-DIMENSIONAL SYMMETRIC FUNCTIONS}

As described by Bunge (1969 and 1982) and Matthies (1987), it is possible to introduce real functions $\overline{\mathrm{K}}_{l}^{\bar{m}}(\mathrm{r})(\bar{m}=-l,-l+1, \ldots, l)$ by means of orthonormalized real combinations of functions (8)

$$
\overline{\mathbf{K}}_{l}^{\bar{m}}(\mathbf{r})=\sum_{m=-l}^{l} \mathbf{A}_{l}^{m, \bar{m}} \mathbf{K}_{l}^{m}(\mathbf{r})
$$

If one denotes $\mathrm{A}_{l}^{m, \bar{m}}$ by

$$
\mathrm{A}_{l}^{m, \bar{m}}=\xi_{\bar{m}}\left[(-1)^{\bar{m}} \delta_{m},-\bar{m} \mid+\eta_{\bar{m}} \delta_{m},{ }_{\mid \bar{m}}\right]
$$

with

$$
\xi_{\bar{m}}=\left\{\begin{array}{l}
\frac{i}{\sqrt{2}} \\
1 \\
\frac{1}{\sqrt{2}}
\end{array} \quad \eta_{\bar{m}}=\left\{\begin{array} { c } 
{ - 1 } \\
{ 0 } \\
{ 1 }
\end{array} \quad \overline { m } \left\{\begin{array}{c}
<0 \\
=0 \\
>0
\end{array}\right.\right.\right.
$$

then, one has

$$
\bar{K}_{l}^{\bar{m}}(\phi, \beta)=\bar{P}_{l}^{|\bar{m}|}(\phi) \begin{cases}\frac{1}{\sqrt{\pi}} \sin (|\bar{m}| \beta) & \bar{m}<0 \\ \frac{1}{\sqrt{2 \pi}} & \bar{m}=0 \\ \frac{1}{\sqrt{\pi}} \cos (|\bar{m}| \beta) & \bar{m}>0\end{cases}
$$

These functions, like (8), form a complete and orthonormalized basis. Therefore, (6) and (7) may be represented by the new expansion

$$
\dot{\mathbf{K}}_{l}^{\mu}(\mathbf{r})=\sum_{\bar{m}=l}^{l} \dot{\mathrm{A}}_{l}^{\bar{m} \mu} \overline{\mathbf{K}}_{l}^{\bar{m}}(\mathbf{r})
$$

with real coefficients $\dot{\mathrm{A}}_{l}^{\bar{m} \mu}$.

Non-cubic Groups

For these point groups, the $\dot{\mathrm{A}}_{l}^{\bar{m} \mu}$ may be chosen quite simple:

$$
\dot{\mathrm{A}}_{l}^{\bar{m} \mu}=\delta_{\bar{m}, \bar{m}^{\prime}}
$$


Values of $\bar{m}^{\prime}$ are given in Tables I and II, as functions of the parameters $l$ and $\mu$. In Table $I$, the location of the symmetry elements have been fitted (for each point group) according to Bunge. For cases where these locations don't agree with other common standards (IEEE Standards on Piezoelectricity, 1978; International Tables of Crystallography, 1983), Table II is also presented.

Table I Selection rules for low-symmetry real two-dimensional functions. Coordinate axes according to Bunge.

\begin{tabular}{|c|c|c|c|c|}
\hline$G$ & $n$ & $l$ & $M(l)$ & $\begin{array}{c}\bar{m}^{\prime}=\bar{m}^{\prime}(l, \mu) \\
\mu=1,2,3 \quad \ldots, M(l)\end{array}$ \\
\hline$C_{\mathrm{n}}$ & $1,2,3,4,6$ & $\forall$ & $2\left[\frac{l}{n}\right]+1$ & \multirow{7}{*}{$\mathrm{n}\left[\mu-\frac{M(l)+1}{2}\right]$} \\
\hline$\underline{\mathrm{C}_{i}}$ & 1 & even & $2 l+1$ & \\
\hline$\underline{C_{s}}$ & 2 & $\forall$ & $l+1$ & \\
\hline$C_{3 t}$ & 3 & even & $2\left[\frac{l}{3}\right]+1$ & \\
\hline \multirow[t]{2}{*}{$S_{4}$} & \multirow[t]{2}{*}{4} & even & $2\left[\frac{l}{4}\right]+1$ & \\
\hline & & odd & $2\left[\frac{l+1}{4}\right]$ & \\
\hline \multirow[t]{3}{*}{$C_{\mathrm{nh}}$} & $2,4,6$ & even & $2\left[\frac{l}{n}\right]+1$ & \\
\hline & \multirow[t]{2}{*}{3} & even & $2\left[\frac{1}{6}\right]+1$ & \multirow[t]{2}{*}{$6\left[\mu-\frac{M(l)+1}{2}\right]$} \\
\hline & & odd & $2\left[\frac{l-2}{6}\right]+2^{*}$ & \\
\hline$C_{\mathrm{nv}}$ & $2,3,4,6$ & $\forall$ & {$\left[\frac{l}{n}\right]+1$} & $\mathrm{n}(\mu-1)$ \\
\hline \multirow[t]{4}{*}{$\overline{D_{\mathrm{n}}}$} & \multirow[t]{2}{*}{$2,4,6$} & even & {$\left[\frac{l}{n}\right]+1$} & \multirow[t]{2}{*}{$(-1)^{l} \mathrm{n}\left[\mu-\frac{\left(1+(-1)^{l}\right)}{2}\right]$} \\
\hline & & odd & {$\left[\frac{l-1}{2}\right]$} & \\
\hline & \multirow[t]{2}{*}{3} & even & {$\left[\frac{l}{3}\right]+1$} & \multirow[t]{2}{*}{$(-1)^{\mu+1} 3\left[\mu-\frac{\left(1+(-1)^{l}\right)}{2}\right.$} \\
\hline & & odd & {$\left[\frac{l}{3}\right]$} & \\
\hline \multirow[t]{3}{*}{$D_{\mathrm{nh}}$} & $2,4,6$ & even & {$\left[\frac{l}{n}\right]+1$} & $\mathrm{n}(\mu-1)$ \\
\hline & \multirow[t]{2}{*}{3} & even & {$\left[\frac{1}{6}\right]+1$} & $6(\mu-1)$ \\
\hline & & odd & {$\left[\frac{1-3}{6}\right]+1 *$} & $3(2 \mu-1)$ \\
\hline
\end{tabular}


Table I Cont'd.

\begin{tabular}{l|c|c|c|c}
\hline$G$ & $n$ & $l$ & $M(l)$ & $\begin{array}{c}\bar{m}^{\prime}=\bar{m}^{\prime}(l, \mu) \\
\mu=1,2,3 \ldots, M(l)\end{array}$ \\
\hline$D_{\text {nd }}$ & 2 & even & {$\left[\frac{l}{4}\right]+1$} & $4(\mu-1)$ \\
\cline { 3 - 5 } & & odd & {$\left[\frac{l-2}{4}\right]+1^{*}$} & $2(2 \mu-1)$ \\
\cline { 2 - 5 } & 3 & even & {$\left[\frac{l}{3}\right]+1$} & $3(\mu-1)$ \\
\hline
\end{tabular}

$* M(1)=0$

Table II Selection rules for low-symmetry real two-dimensional functions. Coordinate axes of selected point groups according to IEEE standards.

\begin{tabular}{l|l|l|l}
\hline$G$ & $l$ & $M(l)$ & $\begin{array}{l}\bar{m}^{\prime}=\bar{m}^{\prime}(l, \mu) \\
\mu=1,2,3 \ldots, M(l)\end{array}$ \\
\hline$C_{2}$ & even & $l+1$ & $\mu-1$ \\
\hline & odd & $l$ & $-\mu$ \\
\hline$C_{\mathrm{s}}$ & $\forall$ & $l+1$ & $\mu-1$ \\
\hline$C_{2 \mathrm{~b}}$ & even & $l+1$ & $(-1)^{\mu-1}[3(\mu-1)]$ \\
\hline$C_{3 \mathrm{v}}$ & $\forall$ & {$\left[\frac{l}{3}\right]+1$} & \\
\hline$D_{3 \mathrm{~d}}$ & even & {$\left[\frac{l}{6}\right]+1$} & $6(\mu-1)$ \\
\hline$D_{3 \mathrm{~b}}$ & even & {$\left[\frac{l-3}{6}\right]+1 *$} & $3(1-2 \mu)$ \\
\cline { 2 - 4 } & odd & {$\left[\frac{l}{4}\right]+1$} & $4(\mu-1)$ \\
\hline$D_{2 \mathrm{~d}}$ & even & {$\left[\frac{l+1}{4}\right]$} & $2(1-2 \mu)$ \\
\cline { 2 - 4 } & odd & & \\
\hline
\end{tabular}

$*_{M}(1)=0$

\section{Cubic Groups}

The characteristic feature of cubic system is their main-diagonal threefold rotation axis.

This symmetry element imposes for the functions (6) or (7) the condition

$$
\dot{\mathbf{K}}_{l}^{\mu}(\phi=\alpha, \beta=0)=\dot{\mathbf{K}}_{l}^{\mu}(\phi=90, \beta=\alpha)
$$


On the other hand, for these groups $m$ is generally even. Therefore if we set

$$
\begin{aligned}
& \dot{\mathrm{A}}_{l}^{\bar{m} \mu}=i \frac{\dot{\mathrm{A}}_{l}^{m \mu}-\dot{\mathrm{A}}_{l}^{-m \mu}}{\sqrt{2}} \bar{m}<0, m>0 \\
& \dot{\mathrm{A}}_{l}^{\bar{m} \mu}=\dot{\mathrm{A}}_{l}^{m \mu} \quad \bar{m}=m=0 \\
& \dot{\mathrm{A}}_{l}^{\bar{m} \mu}=\frac{\dot{\mathrm{A}}_{l}^{m \mu}+\dot{\mathrm{A}}_{l}^{-m \mu}}{\sqrt{2}} \bar{m}>0, m>0
\end{aligned}
$$

then we may establish the function (6) or (7) in real form. Hence, one can see that the functions $\dot{\mathrm{K}}_{l}^{\mu}(\mathrm{r})$ will be linear combinations of the functions $\overline{\mathbf{K}}_{l}^{\bar{m}}(\mathbf{r})$.

\section{Tetrahedral Groups}

The tetrahedral group $T$ has twofold symmetries associated to each of the cubic coordinate axes. The following conditions are valid:

$$
m=2 m^{\prime} ; \dot{\mathrm{A}}_{l}^{-m \mu}=(-1)^{l} \dot{\mathrm{A}}_{l}^{m \mu}
$$

As a consequence, according to (19) we have for the functions (16) that:

for even $l$

$$
\dot{\mathrm{A}}_{l}^{\bar{m} \mu}=\left\{\begin{aligned}
0 & \bar{m}<0 \\
\dot{\mathrm{A}}_{l}^{m \mu} & \bar{m}=m=0 \\
\sqrt{2} \dot{\mathrm{A}}_{l}^{m \mu} & \bar{m}=m>0
\end{aligned}\right.
$$

for odd $l$

$$
\dot{\mathrm{A}}_{l}^{\bar{m} \mu}=\left\{\begin{aligned}
0 & \bar{m}<0 \\
\dot{\mathrm{A}}_{l}^{m \mu} & \bar{m}=m=0 ; \quad m \geq 0 \\
\sqrt{2 i} \dot{\mathrm{A}}_{l}^{m \mu} & \bar{m}=-m<0
\end{aligned}\right.
$$

where the coefficients $\dot{\mathrm{A}}_{l}^{m \mu}$ are calculated from the condition (18).

In the group $T d$, besides the conditions (18) and (20), it must also be true that

$$
\dot{\mathrm{A}}_{l}^{-m \mu}=(-1)^{\frac{m}{2}} \dot{\mathrm{A}}_{l}^{m \mu}
$$

For all $l, \dot{\mathrm{A}}_{l}^{\bar{m} \mu}$ in (21) or (22) are non null when $\bar{m}$ equals $4 m^{\prime}$ or $4 m^{\prime}+2$ respectively.

For group Th, besides the conditions (18) and (20), the selection rule $l=2 l^{\prime}$ must be satisfied. So, for even $l$, its symmetric functions are the same as those for group $T$. 


\section{Octahedral Groups}

Here, the sin terms in equation (6) or (7) must vanish. Thus the symmetric functions (16) may be represented by

$$
\dot{\mathrm{K}}_{l}^{\mu}(\phi, \beta)=\sum_{\bar{m}=0}^{l} \dot{\mathrm{B}}_{l}^{\bar{m}^{\mu}} \overline{\mathrm{P}}_{l}^{\bar{m}}(\phi) \cos \bar{m} \beta
$$

being

$$
\dot{\mathrm{B}}_{l}^{\bar{m} \mu}=\left\{\begin{array}{cc}
\frac{1}{\sqrt{2 \pi}} \dot{\mathrm{A}}_{l}^{0 \mu} & \bar{m}=0 \\
\frac{1}{\sqrt{\pi}} \dot{\mathrm{A}}_{l}^{\bar{m} \mu} & \bar{m} \neq 0
\end{array} \quad ; \bar{m}=4 m^{\prime}\right.
$$

According to (18), these coefficients must satisfy:

$$
\begin{aligned}
& \sum_{\bar{m}=0}^{l} \dot{\mathrm{B}}_{l}^{\bar{m} \mu} \stackrel{+}{a}_{l}^{\bar{m} s}=0 \\
& {\stackrel{+}{{ }_{l}}}^{\bar{m} s}=\left\{\begin{array}{cc}
a_{l}^{\bar{m} s} & s=0 \\
2 a_{l}^{\bar{m} s} & s \neq 0
\end{array},\right.
\end{aligned}
$$

with

$a_{l}^{\bar{m} s}$ are the Fourier coefficients of $\overline{\mathrm{P}}_{l}^{\bar{m}}(\phi)$, thereby either:

or

$$
l=2 l^{\prime} ; \quad s=4 s^{\prime}+2
$$

$$
l=2 l^{\prime}+1 ; \quad s=2 s^{\prime}+1
$$

These coefficients agree with Bunge's (1982) $\dot{\mathrm{B}}_{l}^{{ }^{m \mu}}$ ([001] parallel to Z-axis).

The symmetric functions for group $O_{h}$ are the same as those for $O$ for even $l$.

The real functions obtained in this paragraph fulfill the orthonormality condition (9).

\section{Example}

Suppose a specimen built-up of quartz $\left(D_{3}\right)$ crystallites with triclinic sample symmetry (Bunge and Wenk, 1977). Symmetrized functions (16) corresponding to Table I are reported in Table III and may be expressed explicitly according to (15).

\section{REAL THREE-DIMENSIONAL SYMMETRIC FUNCTIONS}

With the help of coefficients (13) real functions $\overline{\mathrm{T}}_{l}^{\bar{m}, \bar{n}}(\mathrm{~g})$ can also be introduced as orthonormalized real combination of functions $\mathrm{T}_{l}^{m n}(\mathrm{~g})$ [see Bunge (1969 and 1982) and Matthies (1987)] 
Table III Two-dimensional symmetric functions for quartz crystallites-triclinic sample case. ( $1 \leq 4)$.

\begin{tabular}{|c|c|c|c|c|c|c|}
\hline$l$ & $(M l)$ & $N(l)$ & $\mu$ & $\dot{\dot{K}}_{l}^{\mu}(h)$ & $v$ & $\dot{K}_{l}^{v}(y)$ \\
\hline 0 & 1 & 1 & 1 & $\overline{\mathbf{K}}_{0}^{0}$ & 1 & $\overline{\mathbf{K}}_{0}^{0}$ \\
\hline \multirow[t]{5}{*}{2} & 1 & 5 & 1 & $\overline{\mathbf{K}}_{2}^{0}$ & 1 & $\overline{\mathbf{K}}_{2}^{-2}$ \\
\hline & & & & & 2 & $\overline{\mathbf{K}}_{2}^{-1}$ \\
\hline & & & & & 3 & $\overline{\mathbf{K}}_{2}^{0}$ \\
\hline & & & & & 4 & $\overline{\mathbf{K}}_{2}^{1}$ \\
\hline & & & & & 5 & $\overline{\mathbf{K}}_{2}^{2}$ \\
\hline \multirow[t]{7}{*}{3} & 1 & 7 & 1 & $\overline{\mathbf{K}}_{3}^{3}$ & 1 & $\overline{\mathbf{K}}_{3}^{-3}$ \\
\hline & & & & & 2 & $\overline{\mathbf{K}}_{3}^{-2}$ \\
\hline & & & & & 3 & $\overline{\mathbf{K}}_{3}^{-1}$ \\
\hline & & & & & 4 & $\overline{\mathbf{K}}_{3}^{0}$ \\
\hline & & & & & 5 & $\overline{\mathbf{K}}_{3}^{1}$ \\
\hline & & & & & 6 & $\overline{\mathbf{K}}_{3}^{2}$ \\
\hline & & & & & 7 & $\overline{\mathbf{K}}_{3}^{3}$ \\
\hline \multirow[t]{9}{*}{4} & 2 & 9 & 1 & $\overline{\mathbf{K}}_{4}^{0}$ & 1 & $\overline{\mathbf{K}}_{4}^{-4}$ \\
\hline & & & 2 & $\overline{\mathbf{K}}_{4}^{-3}$ & 2 & $\overline{\mathbf{K}}_{4}^{-3}$ \\
\hline & & & & & 3 & $\overline{\mathbf{K}}_{4}^{-2}$ \\
\hline & & & & & 4 & $\overline{\mathbf{K}}_{4}^{-1}$ \\
\hline & & & & & 5 & $\overline{\mathbf{K}}_{4}^{0}$ \\
\hline & & & & & 6 & $\overline{\mathbf{K}}_{4}^{1}$ \\
\hline & & & & & 7 & $\overline{\mathbf{K}}_{4}^{2}$ \\
\hline & & & & & 8 & $\overline{\mathbf{K}}_{4}^{3}$ \\
\hline & & & & & 9 & $\overline{\mathbf{K}}_{4}^{4}$ \\
\hline
\end{tabular}

$$
\overline{\mathrm{T}}_{l}^{\bar{m}, \bar{n}}(\mathrm{~g})=\sum_{m=-l}^{l} \sum_{n=-l}^{l} \mathrm{~A}_{l}^{m, \bar{m}} \mathrm{~A}_{l}^{n, \bar{n}} \mathrm{~T}_{l}^{m n}(\mathrm{~g})
$$

Introducing the real functions

$$
Q_{l}^{m n}(\phi)=i^{m-n} P_{l}^{m n}(\phi)
$$

and keeping in mind the properties of generalised Legendre functions $P_{l}^{m n}(\phi)$ we obtain the explicit expression

$$
\begin{aligned}
\overline{\mathrm{T}}_{l}^{\bar{m}, \bar{n}}\left(\varphi_{1}, \phi, \varphi_{2}\right) & =Q_{l}^{|\bar{m}|,|\bar{n}|}(\phi)\left[C_{1} \cos \left(|\bar{m}| \varphi_{2}+|\bar{n}| \varphi_{1}\right)+C_{2} \sin \left(|\bar{m}| \varphi_{2}+|\bar{n}| \varphi_{1}\right)\right] \\
& +(-1)^{l+|\bar{m}|} Q_{l}^{|\bar{m}|,|\bar{n}|}(\pi-\phi)\left[C_{3} \cos \left(|\bar{m}| \varphi_{2}-|\bar{n}| \varphi_{1}\right)+C_{4} \sin \left\{\left(|\bar{m}| \varphi_{2}-|\bar{n}| \varphi_{1}\right)\right]\right.
\end{aligned}
$$




$$
\begin{aligned}
& +\overline{\mathrm{P}}_{l}^{|\bar{m}|}(\phi)\left[C_{5} \cos \left(|\bar{m}|\left(\varphi_{2}-\frac{\pi}{2}\right)\right)+C_{6} \sin \left(|\bar{m}|\left(\varphi_{2}-\frac{\pi}{2}\right)\right)\right] \\
& +\overline{\mathrm{P}}_{l}^{|\bar{n}|}(\phi)\left[C_{7} \cos \left(|\bar{n}|\left(\varphi_{1}-\frac{\pi}{2}\right)\right)+C_{8} \sin \left(|\bar{n}|\left(\varphi_{1}-\frac{\pi}{2}\right)\right)\right]
\end{aligned}
$$

where the coefficients $C_{l}$ are reported in Table IV.

Functions (31) have the properties

$$
\begin{gathered}
\overline{\mathrm{T}}_{l}^{\bar{m}, \bar{n}}(0,0,0)=S g(\bar{m}) \delta_{\bar{m}, \bar{n}} \\
S g(m)=\frac{\bar{m}}{|\bar{m}|} \\
\overline{\mathrm{T}}_{l}^{\bar{m}, \bar{n}}(0, \pi, 0)=(-1)^{l+|\bar{m}|} \delta_{\bar{m}, \bar{n}}
\end{gathered}
$$

being

and among these and (15) the following relations hold:

$$
\begin{aligned}
& \overline{\mathrm{T}}_{l}^{\bar{m}, 0}\left(\phi, \varphi_{2}\right)=\sqrt{\frac{4 \pi}{2 l+1}} \overline{\mathbf{K}}_{l}^{\bar{m}}\left(\phi, \varphi_{2}-\frac{\pi}{2}\right) \\
& \overline{\mathrm{T}}_{l}^{0, \bar{n}}\left(\varphi_{1}, \phi\right)=\sqrt{\frac{4 \pi}{2 l+1}} \overline{\mathrm{K}}_{l}^{\bar{n}}\left(\phi, \varphi_{1}-\frac{\pi}{2}\right)
\end{aligned}
$$

Moreover these functions, like $\mathrm{T}_{l}^{m n}(\mathrm{~g})$, form a purely real and normalised basis of the generalised spherical harmonics. Therefore, the generalised spherical harmonics (2) which are consistent with both crystal and sample symmetries may be represented by

$$
\dot{\mathrm{T}}_{l}^{\mu v}(\mathrm{~g})=\sum_{m=-l}^{l} \sum_{n=-l}^{l} \dot{\mathrm{A}}_{l}^{\bar{m} \mu} \dot{\mathrm{A}}_{l}^{\bar{n} \nu} \mathrm{T}_{l}^{\bar{m} \bar{n}}(\mathrm{~g})
$$

where the $\dot{\mathrm{A}}_{l}^{\bar{m} \mu}, \dot{\mathrm{A}}_{l}^{\bar{n} v}$ coefficients are those described above.

These symmetric functions fulfill the orthonormality condition (3).

\section{Example}

Now we suppose the cubic-orthorhombic case. The symmetrized functions (36) may be expressed by the expansion

$$
\dot{\mathrm{T}}_{l}^{\mu \nu}(\mathrm{g})=\sqrt{2 \pi} \dot{\mathrm{B}}_{l}{ }^{\mu \mu} \sum_{\pi=-l}^{l} \dot{\mathrm{A}}_{l}^{\bar{n} \nu} \overline{\mathrm{T}}_{l}^{\bar{m} \bar{n}}(\mathrm{~g})+\sqrt{\pi} \sum_{m>0}^{l} \dot{\mathrm{B}}_{l}^{\bar{m} \mu}\left[\sum_{\bar{n}=-l}^{l} \dot{\mathrm{A}}_{l}^{\bar{n} \nu} \mathrm{T}_{l}^{\bar{m} \bar{n}}(\mathrm{~g})\right]
$$

We obtain that the $\dot{\mathrm{B}}_{l}^{\bar{m} \mu}$ coefficients calculated from (26-28) as well the functions reported in Table V, agree with the corresponding entities as given by Bunge (1982), for $l \leq 6$. 
102

O. RAYMOND ET $A L$.

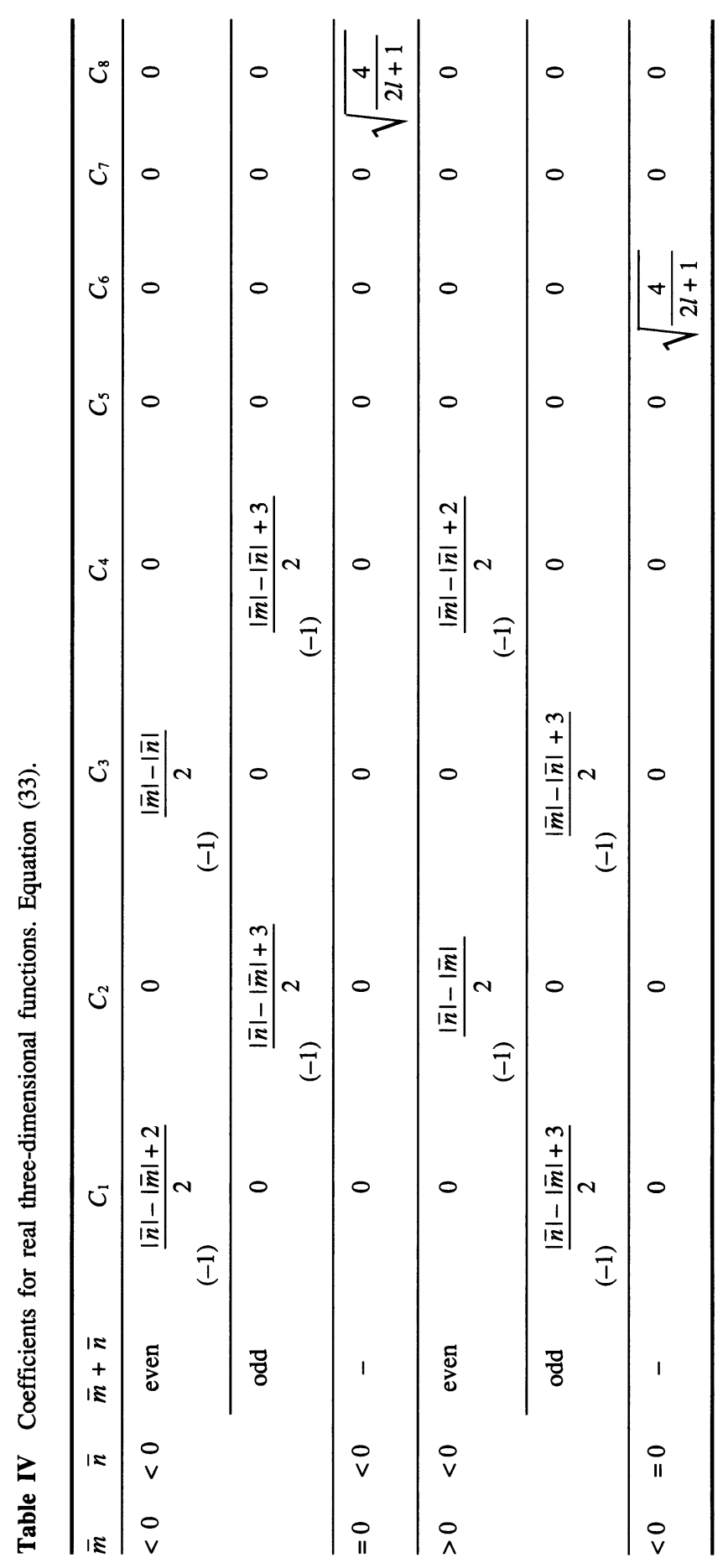


REAL SPHERICAL HARMONICS

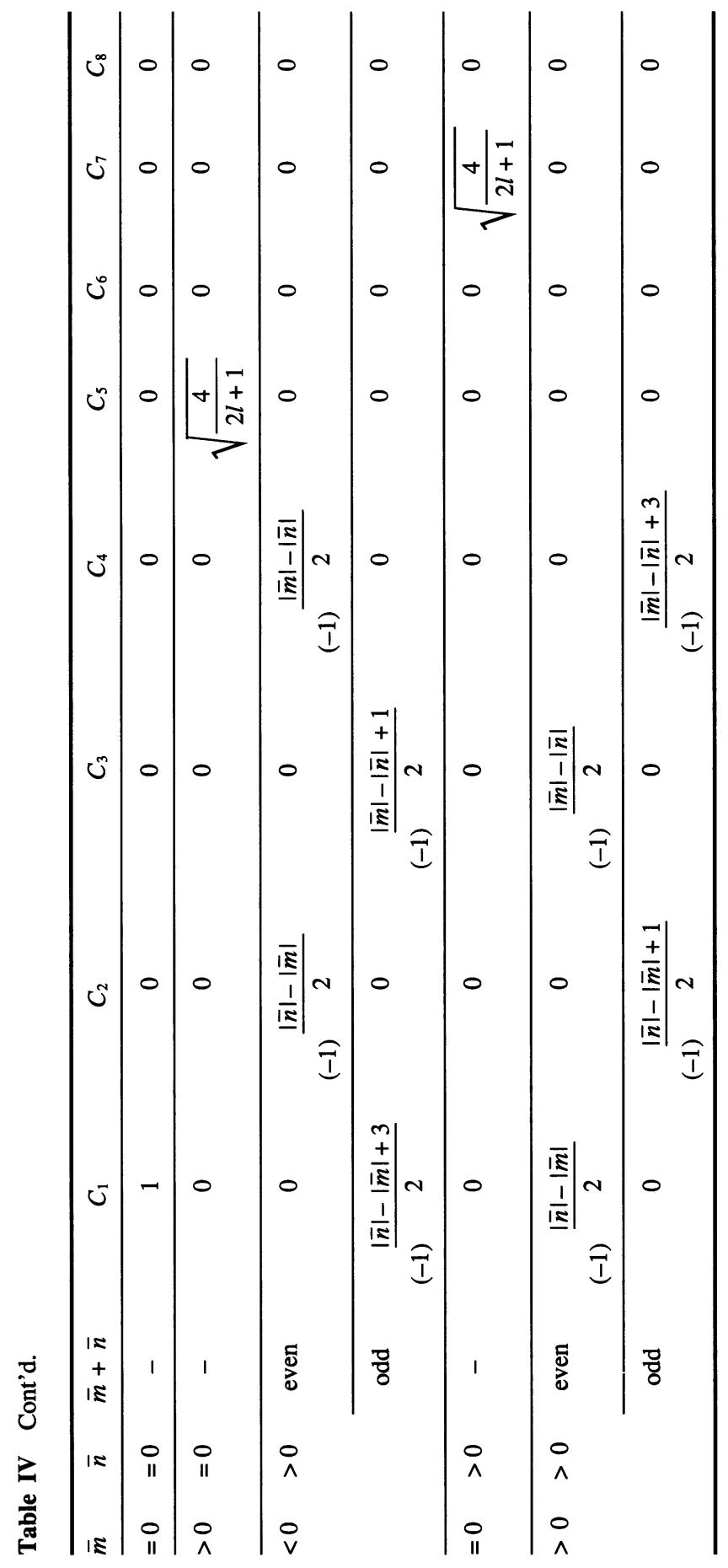


Table V Three-dimensional symmetric functions for cubic-orthorrombic case $(1 \leq 6)$.

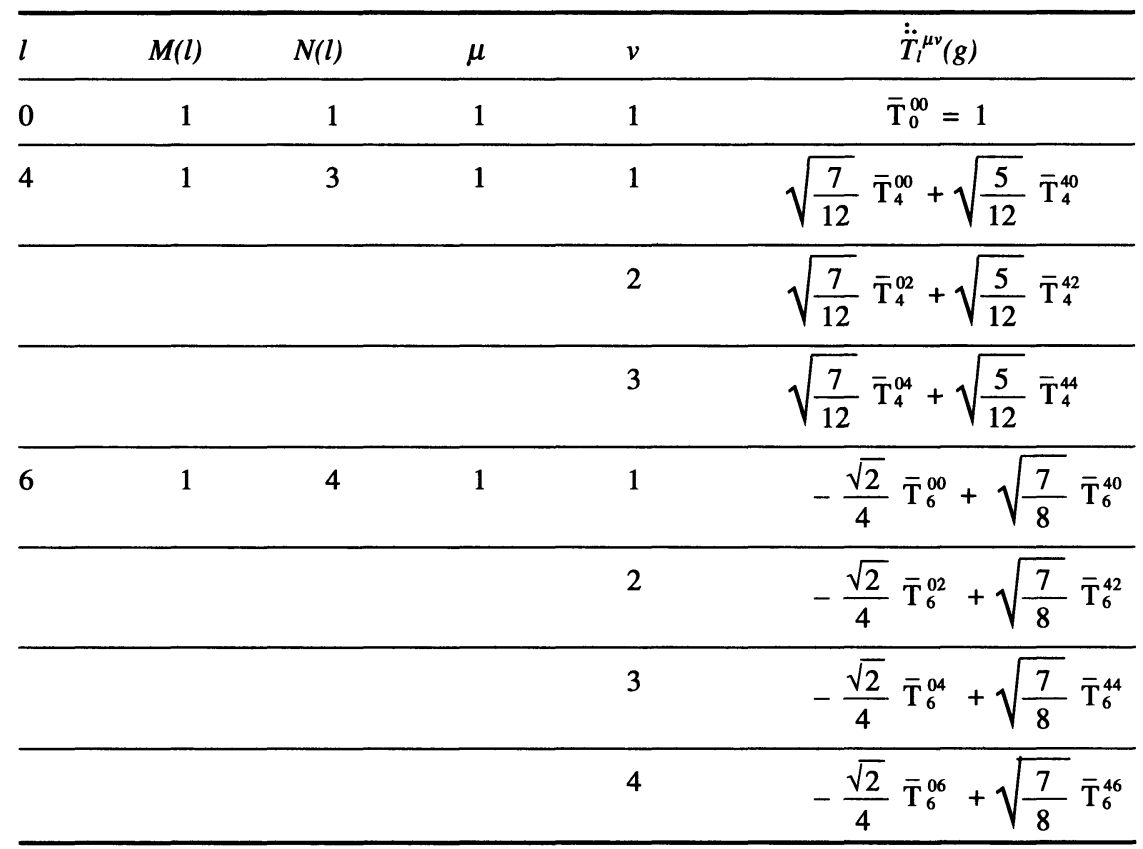

\section{References}

Bunge, H. J. (1969). Mathematische Methoden der Texturanalyse. Akademie Verlag. Berlin.

Bunge, H. J. (1982). Texture Analysis in Material Science. Butterworths, London.

Bunge, H. J. and Wenk, H. R. (1977). Three-dimensional Texture Analysis of Three Quarzites (trigonal crystal and triclinic specimen symmetry). Tectonophysics, 40, 257-285.

Gel'fand, I. M., Minlos, R. A. and Shapiro, Z. Ya. (1963). Representations of the Rotation and Lorentz Groups and their applications. Pergamon Press, Oxford/London/New York/Paris.

IEEE Standards on Plezoelectricity. IEEE Std. 176 (1978).

International Tables of Crystallography (ITC). Reidel Publ. Co., 1983 (Dordrech).

Matthies, S., Vinel, G. W. and Helming, K. (1987). Standard Distributions in Texture Analysis. Vol

1. Akademie Verlag, Berlin. 